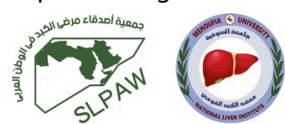

\title{
Etiology and outcome of neonatal cholestasis: an experience in a tertiary center of Bangladesh
}

Salahuddin Mahmud ${ }^{1 *} \mathbb{D}$, Jahida Gulshan ${ }^{2}$, Mashud Parvez ${ }^{3}$, Farhana Tasneem ${ }^{4}$ and Syed Shafi Ahmed ${ }^{5}$

\begin{abstract}
Background: Neonatal cholestasis (NC) is a major cause of morbidity and mortality in young infants. This study examines the etiology of $\mathrm{NC}$ and its outcome during 2 years of follow-up at a tertiary referral center in Bangladesh.

Results: Out of 80 cholestatic infants, $60 \%$ had intrahepatic cholestasis with a mean age of onset of $12.4 \pm 2.8$ days and a mean age of admission of $82.4 \pm 29.0$ days. The remaining $40 \%$ were extrahepatic with a mean age of onset of $6.7 \pm 2.3$ days and a mean age of admission of $94.6 \pm 50.4$ days. Biliary atresia (BA), idiopathic neonatal hepatitis (INH), and TORCH (Toxoplasma, rubella, cytomegalovirus, and herpes simplex) infection except rubella were the most common causes. After receiving treatment, $46.2 \%$ of the cases improved, $23.8 \%$ deteriorated with morbidity, and $30 \%$ died. The majority of the children with INH, TORCH, choledochal cyst, hypothyroidism, galactosemia, and urinary tract infection (UTI) with sepsis were improved. Significant mortality was found in BA (56.6\%), intrahepatic bile duct paucity (PIBD) (100\%), and progressive familial intrahepatic cholestasis (PFIC) (100\%) whereas the rest of BA (43.4\%) live with persistent morbidity. Significant clinical improvement was observed in 37 (46.2\%) cases of cholestasis evidenced by decreasing jaundice, change of color of urine from dark to normal color, change of stool color from pale to yellow, and gradual decrease in liver size from hepatomegaly state. In addition, decreasing median total bilirubin, direct bilirubin, alanine transaminase, gamma-glutamyl transferase, and alkaline phosphatase showed biochemical improvement at 2 years follow-up. The age of admission, etiology, and presence of ascites are the predictors of outcomes.

Conclusion: BA was the most common cause of extrahepatic while INH and TORCH infection were the most common cause of intrahepatic cholestasis. Majority of children with intrahepatic cholestasis improved but deteriorated with BA and genetic causes. Prompt referral and early diagnosis as well as the etiology of NC were the main determinants of the favorable outcome.
\end{abstract}

Keywords: Bangladesh, Child, Neonatal cholestasis, Biliary atresia, Idiopathic neonatal hepatitis, Outcome, Pediatric liver transplant

\section{Background}

Neonatal cholestasis (NC) is a reduced bile formation or flow with retention of biliary substances inside the

\footnotetext{
*Correspondence: drsmbablu@gmail.com

${ }^{1}$ Pediatric Gastroenterology, Hepatology \& Nutrition, Bangladesh Institute of Child Health, Dhaka Shishu (Children) Hospital, Sher-E-Bangla Nagar, Dhaka 1207, Bangladesh

Full list of author information is available at the end of the article
}

liver, which can be presented as conjugated hyperbilirubinemia in the first 90 days of extra-uterine life $[1,2]$. When conjugated serum bilirubin is greater than $20 \%$ of total bilirubin, it is a pathological condition that always requires evaluation [3-7]. Idiopathic neonatal hepatitis (INH) and biliary atresia (BA) are the most commonly observed causes of cholestatic jaundice in the first months of life $[8,9]$. Idiopathic, congenital infection, sepsis, and inborn errors of metabolism are relatively more common causes of neonatal hepatitis compared to toxic 
causes, post-hemolytic states, neonatal acute hepatic necrosis, parenteral nutrition, and chromosomal anomalies as well as familial syndromes [10]. Among Asians, alpha-1 antitrypsin ( $\alpha 1 \mathrm{AT})$ deficiency is uncommon but common among Caucasians [11].

A palpable mass in the right upper quadrant may indicate a choledochal cyst (CC). The dysmorphic facial features can suggest syndromic and chromosomal (Down, Alagille, etc.) disorders [4]. A low or normal GGT suggests the presence of progressive familial intrahepatic cholestasis (PFIC type1 or PFIC type 2) [4, 7, 12-15]. Abdominal ultrasonography in BA includes the triangular cord sign with non-visualized or contracted gall bladder [4-7]. Since hepatobiliary scintigraphy (HS) is not only expensive and time-consuming but also poorly specific, many centers do not routinely use this test [16-19]. Liver biopsy (LB) is considered as one of the most reliable investigations for the diagnosis of BA, with an accuracy of $88.2-96.9 \%$ [20, 21]. The gold standard for the diagnosis of BA is a per-operative cholangiogram which has a diagnostic accuracy of $100 \%[1,12,20,21]$.

Ursodeoxycholic acid (UDCA), cholestyramine, and/or phenobarbital can play a major role in maintaining bile flow as well as management of pruritus [7, 12, 13, 22-24].
Kasai hepatoportoenterostomy (KPE) within due time is a successful bridging surgery of BA [12, 22-24]. Unfortunately, with no opportunity for pediatric LT, many cholestatic cases struggle in the country to reach a favorable outcome [11, 25].

There is no comprehensive and authentic data regarding the etiology and outcome of $\mathrm{NC}$ especially in the pediatric population of Bangladesh. This study was undertaken to assess the outcome of NC among Bangladeshi infants.

\section{Methods \\ Study design}

A retrospective cohort study was done in the Department of Pediatric Gastroenterology, Hepatology \& Nutrition, Dhaka Shishu (Children) Hospital, Dhaka, Bangladesh, among 80 infants admitted to the hospital from July 2014 to June 2016 with cholestatic jaundice and were followed up monthly for a minimum of 2 years.

A study flow chart is added here for better understanding (Fig. 1).

The study included, irrespective of gender, a total of 80 admitted cases of NC with the age of onset less than 1 year and meeting at least one of the following inclusion

Sample: Infants with cholestatic jaundice admitted to the hospital from

July 2014 to June 2016 with a minimum follow-up of two years (till June

2018) on a monthly basis. $(n=80)$

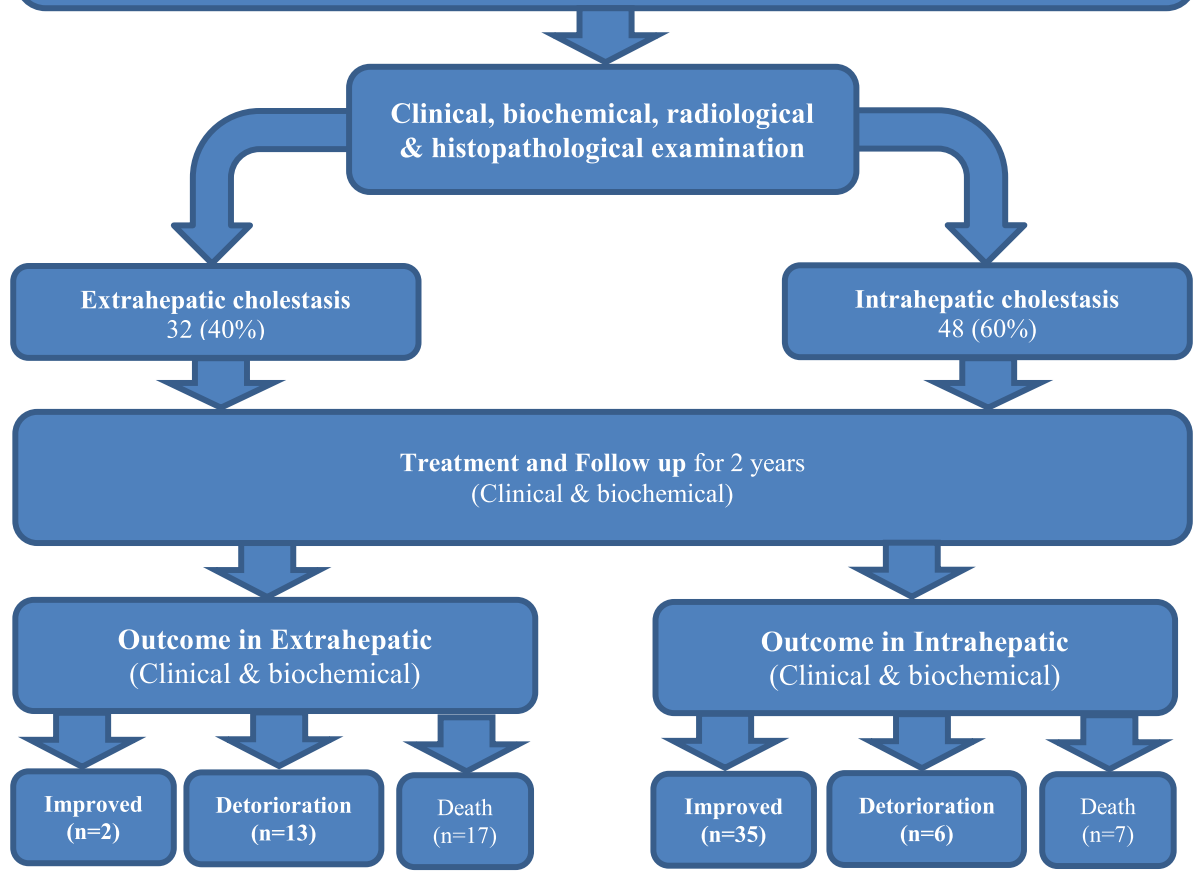

Fig. 1 Study flow chart 
criteria: (i) conjugated bilirubin was more than $1 \mathrm{mg} /$ $\mathrm{dl}$, (ii) total serum bilirubin was less than or equal to 5 $\mathrm{mg} / \mathrm{dl}$, and (iii) conjugated bilirubin was higher than $20 \%$ of total serum bilirubin when total serum bilirubin was more than $5 \mathrm{mg} / \mathrm{dl}$. Children with Jaundice due to other reasons than cholestasis were excluded from the study. Clinical history, physical examination, investigation results, and treatments given were recorded. The outcome was recognized by identifying improvement, deterioration with morbidity, or mortality based on various clinical and biochemical parameters.

\section{Laboratory tests}

Complete blood count $(\mathrm{CBC})$ with culture and liver function tests [S. bilirubin total, direct, indirect, ALT, ALP, GGT, and prothrombin time (PT)] were done. Urine was tested for non-glucose reducing substances (NGRS) and bacterial culture. Both fasting and after a feed ultrasonography of hepatobiliary system (HBS) was done in all cases to detect polysplenia or asplenia and CC. HIDA scan was done from Institute of Nuclear Medicine, Bangabandhu Sheikh Mujib Medical University (BSMMU), after administration of phenobarbitone $(5 \mathrm{mg} / \mathrm{kg} /$ day orally in two divided doses for at least 5 days). At scintigraphy, the absence of radioactivity in the small bowel after $24 \mathrm{~h}$ was taken as absent tracer excretion. However, 21 (26.3\%) cases were excluded from this examination due to long queues and transient unavailability of tracer elements. LB was done with the help of Automated Biopsy Gun $(18 \mathrm{G} \times 16 \mathrm{~cm}$, PD $22 \mathrm{~mm})$ under local anesthetics (2\% lidocaine) and intravenous midazolam $(0.1$ $\mathrm{mg} / \mathrm{kg}$ ) with $3 \mathrm{~h}$ fasting followed by an immediate fixation of the biopsy specimens in 10\% formal saline. Biopsy was not done in patients with huge ascites, coagulopathy, and lack of parental consent [40 (50\%)].

\section{Disease specific diagnosis}

BA cases in our study satisfied all four clinical criteria (1) persistent acholic stools, (2) specific sonography features (rudimentary gall bladder or triangular cord sign), (3) non-excretory scintigraphy, and (4) suggestive liver histology (bile ductular proliferation, portal tract fibrosis, expanded portal tracts, and bile plugs in portal triads). In cases where the ultrasonogram (USG) report was suggestive of CC or non-conclusive, a magnetic resonance cholangiopancreatography (MRCP) confirmed the diagnosis.

To identify the intrahepatic causes of cholestasis, TORCH screening, thyroid function test, and HBsAg had been done. When the cytomegalovirus (CMV) was IgM positive or IgG more than 10 fold than normal then urinary CMV PCR (polymerase chain reaction) had been done. Karyotyping had been done in one suspected case of Down syndrome and galactose-1-phosphate uridylyltransferase (GALT) assay only in NGRS-positive cases. Free thyroxine (FT4) and thyroid-stimulating hormone (TSH) had been tested for the diagnosis of congenital hypothyroidism. The paucity of intrahepatic bile duct (PIBD) had been diagnosed by loss of intrahepatic bile ducts in more than $50 \%$ of portal tracts in a specimen that contained at least 10 portal tracts. PIBD was syndromic or non-syndromic was determined by echocardiogram, ophthalmological examination, and X-ray thoracolumbar spine both views. PFIC (type I/II) had been diagnosed by low or normal GGT due to the unavailability of DNA testing in Bangladesh. Although low or normal GGT can also be present in inborn errors of bile acid synthesis or metabolism, serum bile acid assay, and genetic testing could not be done due to unavailability in Bangladesh. Any condition was termed as INH when all test results were insignificant for any condition.

\section{Treatment protocol}

Once a final diagnosis was achieved, in all cases cholestasis, the child was treated with medium chain triglyceride (MCT) enriched coconut oil with breast milk (except galactosemia), phenobarbital, UDCA and/or cholestyramine, fat-soluble vitamins (vitamin-A, D, E, K), water-soluble vitamins, calcium, zinc, and folic acid. KPE within due time (only in less than 3 months of age with informed written consent) for BA and surgical excision of $\mathrm{CC}$ had been done. Kasai procedure was considered to be successful if it resulted in the restoration of bile flow and normalization of serum bilirubin (less than $20 \mu \mathrm{mol} / \mathrm{L}$ ) at any time. 26 For CMV infection, oral Valganciclovir in a dose of $16 \mathrm{mg} / \mathrm{kg} /$ day was given 12 hourly for up to 6 months. For HSV infection, an injectable form of Acyclovir $20 \mathrm{mg} / \mathrm{kg} / \mathrm{dose}$ was given 8 hourly for up to 14 days. As Sulfadiazine was not available in our country, only Pyrimethamine and Leucovorin for 1 year were added for toxoplasmosis. Thyroxine hormone therapy in a dose of $5-10 \mu \mathrm{gm} / \mathrm{kg} /$ day was used for hypothyroidism, lactosefree milk with MCT enriched coconut oil was given for galactosemia, and appropriate antibiotics for 7-10 days were given according to culture and sensitivity report of the urine and blood for urinary tract infection (UTI) and sepsis.

\section{Follow-up}

Basic clinical information including the growth parameters and present liver status and its complications were noted during monthly follow-ups. The outcome measures had been classified as improvement, deterioration (morbidity), or mortality based on the clinical reviews and biochemical findings. The age at death (months) was noted. Morbidity included poor general health, failure to thrive (-2SD for weight for age), the presence of chronic 
liver disease (CLD), portal hypertension (PH), liver cirrhosis, liver failure or its complications, and the need for LT.

\section{Statistical analysis}

Data were collected from the official medical records of the children and were analyzed with the Statistical Package for Social Science (SPSS) version 24.

\section{Results}

\section{Basic features of the reported cases}

The mean age of onset of the overall cohort was 10.23 days and ranging from 3 to 17 days. Although the mean age of onset of intra and extrahepatic cases were significantly different ( $p$ value $<0.001$ ), there was no significant difference between the mean age of admission of the two groups ( $p$ value 0.09) (Fig. 2).

The antenatal, natal, and postnatal history of the cholestatic infants were significantly different in the two groups. Maternal fever, maternal rash, and presence of consanguinity were significantly less in extra-hepatic cases ( $p$ value $=0.002,<0.001$, and $<0.001$, respectively) (Table 1).

A significant difference ( $p$ value $=0.002)$ was observed between the male to female ratio of the intrahepatic group (male to female=1.6:1) and the extrahepatic group (male to female $=1: 2.2$ ). Intra-hepatic cholestatic cases were more frequently observed among pre-term (66.6\%) children and extra-hepatic cholestasis was more frequent among full-term children $(p$ value $<0.001)$.

A summary of the findings on clinical presentation of the infants are presented in Table 2.

Persistent pale stool was more in extra-hepatic cases, and intermittent pale stool was predominant in
Table 1 Ante-natal, natal, and post-natal history of cholestatic infants

\begin{tabular}{|c|c|c|c|}
\hline & $\begin{array}{l}\text { Extra-hepatic } \\
\text { cholestasis } \\
(n=32)\end{array}$ & $\begin{array}{l}\text { Intra-hepatic } \\
\text { cholestasis } \\
(n=48)\end{array}$ & $P$ value \\
\hline \multicolumn{4}{|l|}{ Maternal history } \\
\hline Fever & $4(12.5 \%)$ & $16(35.3 \%)$ & 0.011 \\
\hline Rash & $01(3.1)$ & $22(45.8 \%)$ & 0.000 \\
\hline Pruritus & $01(3.1)$ & $6(12.5 \%)$ & 0.051 \\
\hline \multicolumn{4}{|l|}{ Family (consanguinity) } \\
\hline Present & $00(0.0 \%)$ & $10(20.8 \%)$ & 0.000 \\
\hline Absent & $32(100 \%)$ & $38(79.2 \%)$ & \\
\hline \multicolumn{4}{|l|}{ Infants } \\
\hline Age of onset (days) & $6.8 \pm 2.3$ & $12.4 \pm 2.8$ & 0.000 \\
\hline $\begin{array}{l}\text { Age of admission } \\
\text { (days) }\end{array}$ & $99.6 \pm 52.67$ & $82.4 \pm 29$ & 0.099 \\
\hline \multicolumn{4}{|l|}{ Sex } \\
\hline Male & $10(31.3 \%)$ & $30(62.5 \%)$ & 0.002 \\
\hline Female & $22(68.8 \%)$ & $18(37.5 \%)$ & \\
\hline \multicolumn{4}{|l|}{ Gestational age } \\
\hline Term & $28(87.5 \%)$ & $16(33.3 \%)$ & 0.000 \\
\hline Pre-term & $4(12.5)$ & $32(66.6 \%)$ & \\
\hline
\end{tabular}

intra-hepatic cases ( $p$ value $<0.001$ for both). No significant difference was observed among the two groups for the other clinical characteristics (Table 2).

The results of the liver function tests of cholestatic infants are shown in Table 3.

Serum total, direct bilirubin, ALT, alkaline phosphatase, and GGT were higher than the normal range for both the cholestatic groups. There was no significant difference between the serum total of the two groups as well as the direct bilirubin. ALT was significantly greater

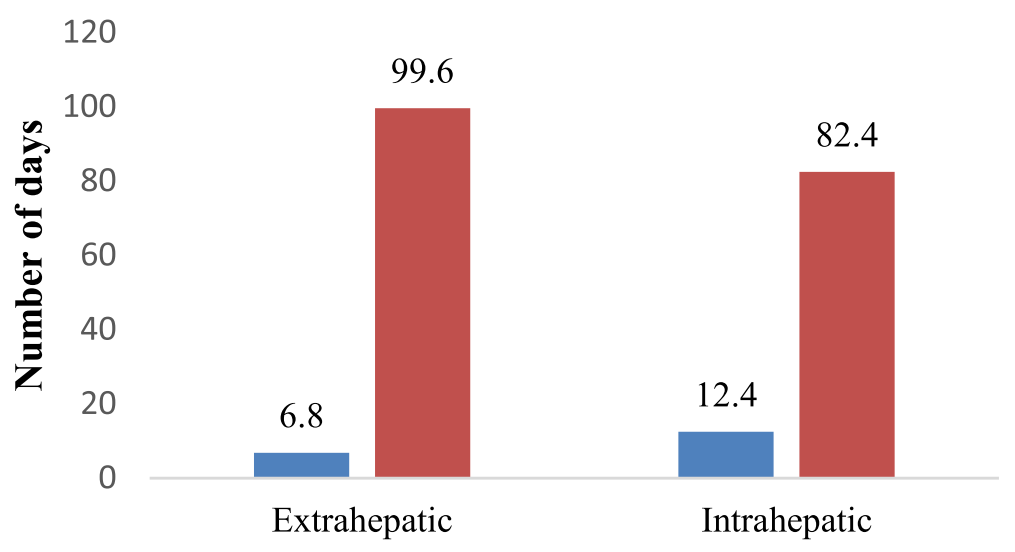

Age of onset Age of admission

Fig. 2 Comparison of the mean age of onset and the mean age of admission 
Table 2 Clinical presentation of cholestatic infants

\begin{tabular}{llll}
\hline Clinical characteristics & $\begin{array}{l}\text { Extra-hepatic } \\
\text { cholestasis } \\
(\boldsymbol{n}=\mathbf{3 2})\end{array}$ & $\begin{array}{l}\text { Intra-hepatic } \\
\text { cholestasis } \\
(\boldsymbol{n}=\mathbf{4 8})\end{array}$ & $\boldsymbol{P}$ value \\
\hline Jaundice & $32(100 \%)$ & $48(100 \%)$ & $\ldots \ldots$. \\
Dark urine & $24(75 \%)$ & $29(60.4 \%)$ & 0.176 \\
Persistent pale stool & $27(84.3 \%)$ & $11(22.9 \%)$ & $\mathbf{0 . 0 0 0}$ \\
Intermittent pale stool & $05(15.6 \%)$ & $37(77.1 \%)$ & $\mathbf{0 . 0 0 0}$ \\
Hepatomegaly & $27(84.4 \%)$ & $44(91.7 \%)$ & 0.311 \\
Splenomegaly & $18(56.2 \%)$ & $36(75 \%)$ & 0.079 \\
Ascites & $03(9.3 \%)$ & $07(14.6 \%)$ & 0.620 \\
\hline
\end{tabular}

Table 3 Liver function tests of cholestatic infants

\begin{tabular}{llll}
\hline Liver function tests & $\begin{array}{l}\text { Extra-hepatic } \\
\text { cholestasis } \\
(\boldsymbol{n}=\mathbf{3 2})\end{array}$ & $\begin{array}{l}\text { Intra-hepatic } \\
\text { cholestasis } \\
(\boldsymbol{n}=\mathbf{4 8})\end{array}$ & $\boldsymbol{P}$ value \\
\hline S. total bilirubin (mg/dl) & $8.26 \pm 3.49$ & $12.72 \pm 1.49$ & 0.176 \\
Direct bilirubin (mg/dl) & $4.40 \pm 1.76$ & $3.78 \pm 0.73$ & 0.076 \\
ALT (U/L) & $160.62 \pm 66.18$ & $459.08 \pm 59.10$ & $\mathbf{0 . 0 0 0}$ \\
Alkaline phosphatase & $786.87 \pm 122.85$ & $317.50 \pm 70.00$ & $\mathbf{0 . 0 0 0}$ \\
(U/L) & & & \\
GGT (U/L) & $886.87 \pm 122.85$ & $212.60 \pm 42.68$ & $\mathbf{0 . 0 0 0}$ \\
INR & $1.18 \pm 0.12$ & $1.53 \pm 0.28$ & $\mathbf{0 . 0 0 0}$ \\
\hline
\end{tabular}

Table 4 Liver biopsy findings of studied subjects ( $n=40$ )

\begin{tabular}{|c|c|c|}
\hline Findings & Diagnosis & Frequency (\%) \\
\hline Ductular proliferation, prominent bile plug, marked portal fibrosis & Biliary atresia & $24(60 \%)$ \\
\hline $\begin{array}{l}\text { Disruption of normal liver architecture, chronic inflammatory cell infiltration, giant } \\
\text { cell transformation }\end{array}$ & Idiopathic neonatal hepatitis & $14(35 \%)$ \\
\hline Paucity of interlobular biliary tract & Intra-hepatic bile duct paucity & $2(5 \%)$ \\
\hline
\end{tabular}

in intra-hepatic cases. Alkaline phosphatase and GGT were significantly higher in extra-hepatic cases $(p$ value $<$ $0.001)$. INR was normal in extra-hepatic cases but slightly raised in intra-hepatic cases $(p$ value $<0.001)$.

Hepatobiliary scintigraphy was positive in all infants of extra-hepatic causes because of lack of parental consent, coagulopathy, huge ascites etc.

Liver biopsy was done only in 40 out of 80 cases (Table 4 and Fig. 3).

\section{Etiology}

Overall speaking, BA was the major cause of cholestasis followed by INH and TORCH infections. However, for extrahepatic cases, BA was the most common cause followed by CC. For intrahepatic cases, most common causes were INH and TORCH infections. Each of hypothyroidism, galactosemia, and PIBD were found in (4.1\%) cases. Each of Down syndrome with hypothyroidism, UTI with sepsis and PFIC were found in $2.0 \%$ cases (Fig. 4).

Among 19 TORCH infections, CMV was responsible for nearly $70 \%$ of the cases followed by CMV with HSV co-infection (21.1\%) and toxoplasmosis (10.5\%) (Fig. 5).

\section{Treatment outcomes}

Out of the 80 children under study, a total of 37 (46.2\%) cases improved, 19 (23.8\%) deteriorated, and 24 (30\%) died. A further examination revealed a significant difference between the treatment outcomes of intrahepatic and extrahepatic group ( $p$ value $<0.001)$. Among the extrahepatic cholestatic cases, only $6.3 \%$ improved, $40.6 \%$ deteriorated, and $53.1 \%$ died. On the other hand, among the intrahepatic cholestatic cases, $72.9 \%$ cases improved, $12.5 \%$ deteriorated, and 14.6\% died (Fig. 6).

\section{Disease-specific outcome}

Significant difference was observed in treatment outcome among children with different etiology for both intrahepatic and extrahepatic group ( $p$ value $<0.001)$. All the children with BA either deteriorated or died. 


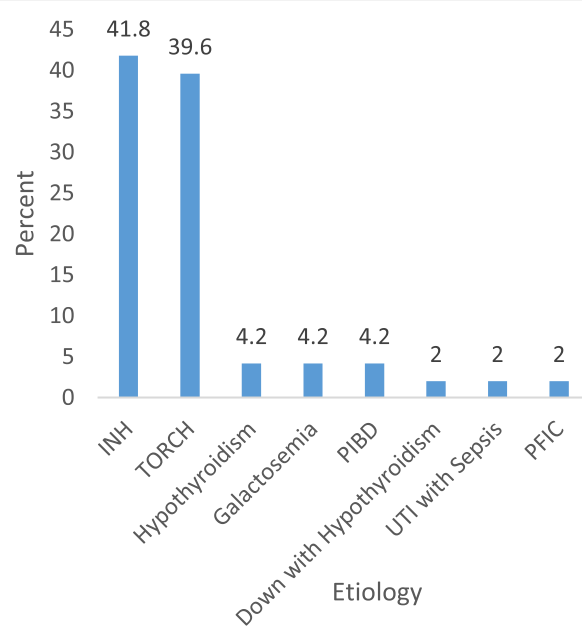

(a) Etiology of Intrahepatic cholestasis

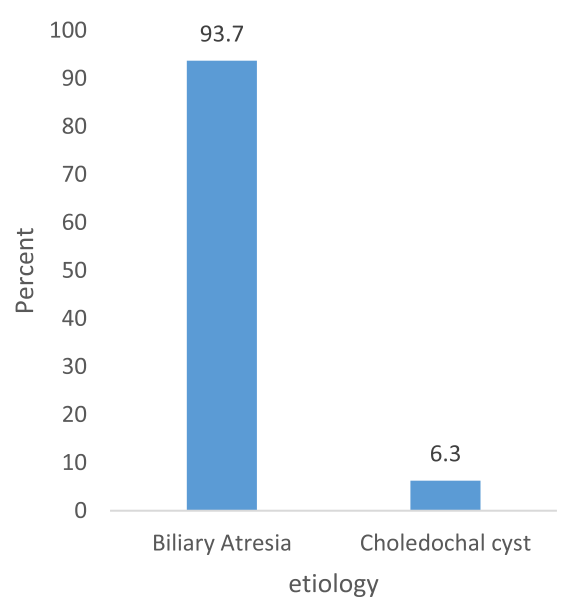

(b) Etiology of Extrahepatic cholestasis

Fig. 4 Etiology of extrahepatic and intrahepatic cholestasis $(n=80)$

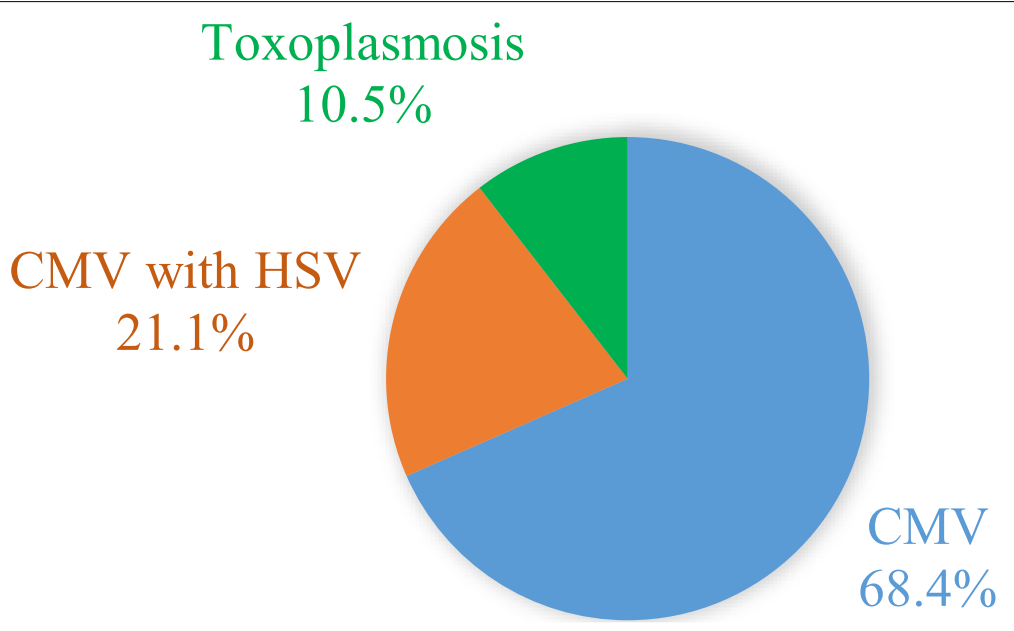

Fig. 5 Responsible viruses in TORCH infection $(n=19)$

The leading cause of death in the extrahepatic group was observed for children with BA [17 (56.6\%)] due to liver cirrhosis with failure. A very high morbidity rate [13(43.4\%)] was also recorded for the same. A significant improvement was seen in CC [2(100\%)].

All the cases of hypothyroidism, Down syndrome with hypothyroidism, galactosemia, and UTI with sepsis (100\%) improved. Significant improvements were also observed in $16(80 \%)$ cases of INH and $13(68.4 \%)$ cases of TORCH (11 cases of CMV \& 2 cases of CMV with HSV) infection. All the cases of PIBD, and PFIC and 3 (15.8\%) cases of TORCH (2 cases of toxoplasmosis and 1 case of CMV with HSV) infection died due to liver cirrhosis with failure. On the other hand, $15 \%$ of INH and $15.8 \%$ of TORCH infections (2 cases of CMV and 1 case of CMV with HSV) gradually developed CLD with PH.

\section{Clinical and biochemical improvement after treatment}

Clinical characteristics after the treatment was recorded for 37 children (35 intrahepatic and 2 extrahepatic cases) and were compared with the same at admission (Table 5).

Significant clinical improvement $(p$ value $<0.001)$ was observed in little less than half of the cholestatic cases. Among 35 intrahepatic cases percentage of children having jaundice, dark urine, pale stool, and hepatomegaly 


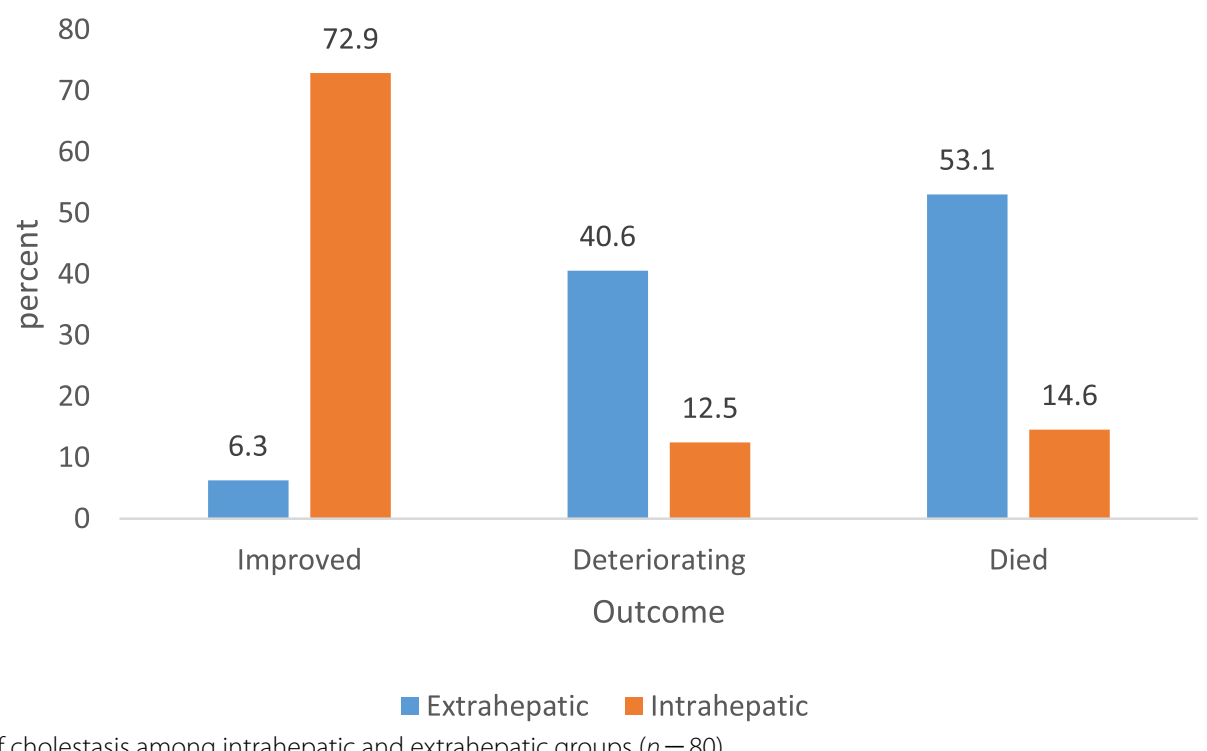

Fig. 6 Outcome of cholestasis among intrahepatic and extrahepatic groups $(n=80)$

Table 5 Clinical and biochemical features of the children before and after treatment

\begin{tabular}{|c|c|c|c|c|c|}
\hline Type & Observed clinical characteristic & $\begin{array}{l}\text { Mean or proportion } \\
\text { (frequency) (before } \\
\text { treatment) }\end{array}$ & $\begin{array}{l}\text { Mean or proportion } \\
\text { (frequency) (after } \\
\text { treatment) }\end{array}$ & Test statistic & $P$ value \\
\hline \multirow[t]{9}{*}{ Intrahepatic $(n=35)$} & Jaundice & $100 \%(35)$ & $11 \%(4)$ & 16.23 & 0.000 \\
\hline & Dark urine & $54 \%(19)$ & $6 \%(2)$ & 5.67 & 0.000 \\
\hline & Pale stool & $71 \%(25)$ & $9 \%(3)$ & 7.59 & 0.000 \\
\hline & Hepatomegaly & $91 \%(32)$ & $14 \%(5)$ & 10.71 & 0.000 \\
\hline & Total bilirubin (mg/dL) & 12.94 & 1.25 & 37.86 & 0.000 \\
\hline & Direct bilirubin (mg/dL) & 3.89 & 0.16 & 25.70 & 0.000 \\
\hline & ALT (IU/L) & 459.66 & 34.29 & 35.98 & 0.000 \\
\hline & Alk Ph (IU/L) & 323.86 & 316.40 & 0.319 & 0.752 \\
\hline & GGT (IU/L) & 203.00 & 51.89 & 13.34 & 0.000 \\
\hline \multirow[t]{9}{*}{ Extrahepatic $(n=2)$} & Jaundice & $100 \%(2)$ & $0 \%(0)$ & \multirow{9}{*}{\multicolumn{2}{|c|}{$\begin{array}{l}\text { Inadequate data to con- } \\
\text { duct test. }\end{array}$}} \\
\hline & Dark urine & $100 \%(2)$ & $0 \%(0)$ & & \\
\hline & Pale stool & $0 \%(0)$ & $0 \%(0)$ & & \\
\hline & Hepatomegaly & $100 \%(2)$ & $0 \%(0)$ & & \\
\hline & Total bilirubin (mg/dL) & 6.3 & 1.2 & & \\
\hline & Direct bilirubin (mg/dL) & 3.3 & 0.05 & & \\
\hline & ALT (IU/L) & 85 & 95 & & \\
\hline & Alk Ph (IU/L) & 870 & 229 & & \\
\hline & GGT (IU/L) & 970 & 121.5 & & \\
\hline
\end{tabular}

reduced significantly. Significant biochemical improvement ( $p$ value $<0.001$ ) was observed by decreasing average total bilirubin, direct bilirubin, ALT, and GGT (Table 5).

The clinical and biochemical test results were observed for only 2 extrahepatic cases, and hence, we abstain from making any comment regarding significant changes in the characteristics of extrahepatic group.

\section{Morbidity and mortality}

A total of 19 (23.8\%) children deteriorated with various morbidity and 24 (30\%) died due to liver cirrhosis with failure (Table 6).

\section{Relationship between age of onset, age of admission,} and treatment outcome

There was no significant difference between the age of onset of the disease among the varying outcomes for either of the two groups ( $p$ value $>0.10)$. 
Table 6 Morbidity and mortality among children with neonatal cholestasis $(n=43)$

\begin{tabular}{lll}
\hline Disease & Morbidity $(\boldsymbol{n = 1 9 )}$ & Cause of death $(\boldsymbol{n}=\mathbf{2 4 )}$ \\
\hline Biliary atresia & $\begin{array}{l}\text { Liver cirrhosis }(n=7) \\
\text { Liver cirrhosis with failure }(n=3) \\
\text { Liver cirrhosis with sepsis }(n=3)\end{array}$ & $\begin{array}{l}\text { Liver cirrhosis with failure }(n=10) \\
\text { Liver cirrhosis with failure with sepsis }(n=7)\end{array}$ \\
INH & $\operatorname{CLD}(n=1)$ & Liver cirrhosis with failure $(n=1)$ \\
& $\operatorname{CLD}$ with $\mathrm{PH}(n=1)$ & \\
TORCH & $\mathrm{Cirhosis}(n=1)$ & Liver cirrhosis with failure $(n=2)$ \\
Intrahepatic bile duct paucity & $\mathrm{CLD}(n=2)$ & Liver cirrhosis with sepsis $(n=1)$ \\
PFIC & $\mathrm{CLD} \mathrm{with} \mathrm{PH}(n=1)$ & Liver cirrhosis with failure $(n=2)$ \\
\hline
\end{tabular}

However, a delayed age of presentation, referral, and diagnosis was found to have significant impact on outcomes. The infants who were admitted within two months $(59.6 \pm 30.0$ days) of age with cholestasis had favorable outcome in relation to non-improved cases with delayed admission ( $p$ value $<0.001)$. A further analysis showed a significant difference between the mean age of admission of the improved and nonimproved cases for both the intrahepatic and extrahepatic groups ( $p$ values $=0.02$ and 0.001 , respectively) . Mean age of admission was much earlier in improved cases in relation to non-improved (Fig. 7).

Gender of children, consanguinity, and treatment outcome Gender of children had no significant association with treatment outcome for either of the two groups ( $p$ value $=0.334$ for intrahepatic and 0.466 for extrahepatic group, respectively). Consanguinity was absent among extrahepatic cases and was not significantly associated with treatment outcome for intrahepatic cases ( $p$ value $=0.79$ ).

\section{Ascites and treatment outcome}

Ascites had a significant association with the treatment outcome in the intrahepatic group ( $p$ value $<0.01$ ). However, out of 80 children, ascites was present in only 10 (7 in the intrahepatic group and 3 in the extrahepatic group) and $100 \%$ of them either deteriorated or died.

\section{Discussion}

A family history of consanguinity is very important for galactosemia, tyrosinemia, PFIC, and Alagille syndrome as all are autosomal recessive disorders. This study observed the presence of consanguinity in $20.8 \%$ of the intrahepatic cholestatic cases, but the absence of the same in extra-hepatic cholestatic cases. Nahid et al., in an earlier study on Bangladesh, also observed the

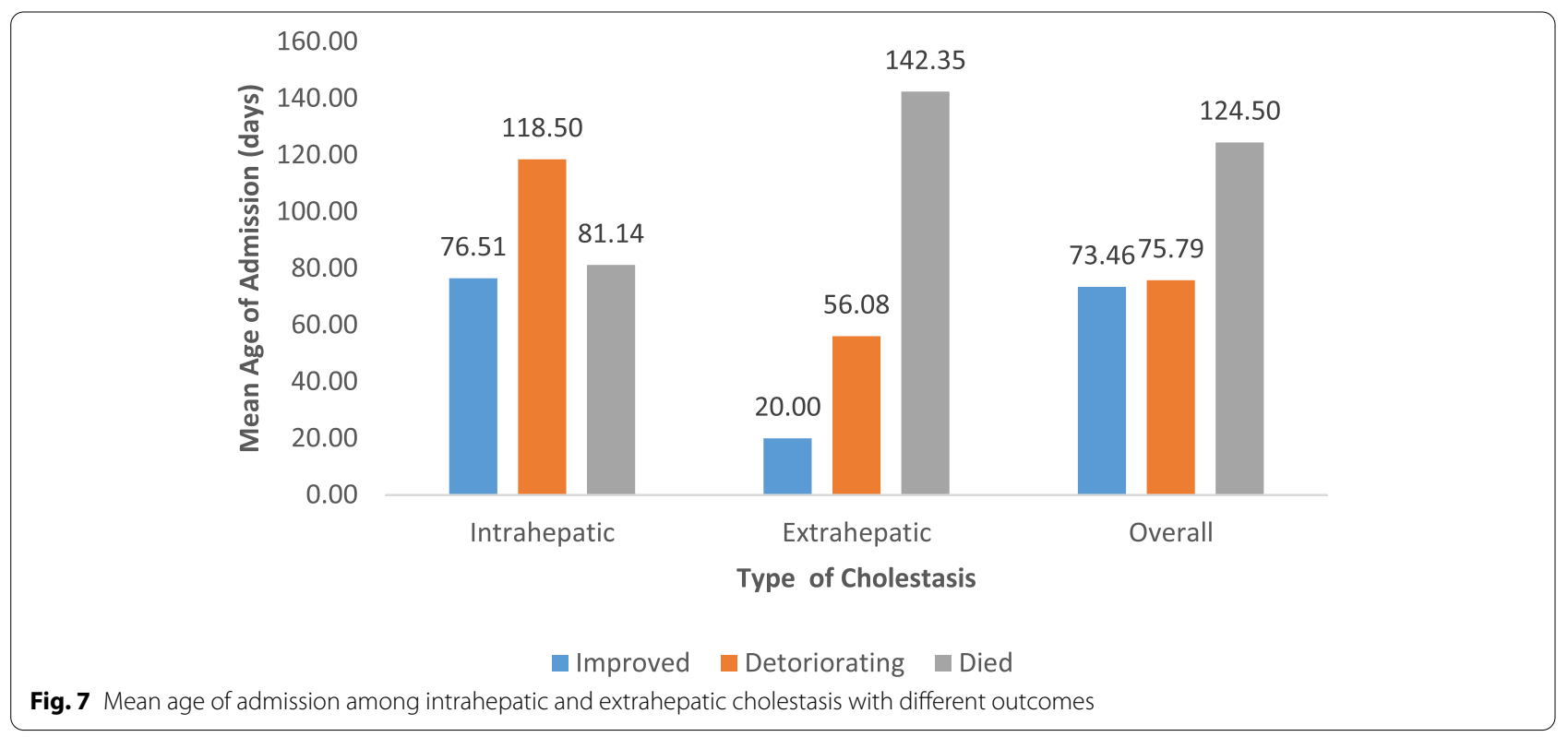


presence of consanguinity in $16.7 \%$ of the intrahepatic cases [26].

Similar to the earlier studies on Bangladesh, this study identifies BA, INH, \& TORCH infections as the major three causes of $\mathrm{NC}[26,27]$. Isolated hypothyroidism and hypothyroidism with Down syndrome are common in intrahepatic cases and some endocrine, metabolic, and genetic disorders might be present with intrahepatic cholestasis $[24,26,27]$ and our study observed similar findings.

The lower percentage of galactosemia among intrahepatic cholestasis in the present study (as compared to the earlier studies on Bangladesh [26, 27]) might be a result of the usage of recent investigational approach like enzyme assay in the study. A reduced percentage of UTI with sepsis as compared to earlier studies [27] is probably an evidence of increased awareness of parents as well as primary health care workers regarding early diagnosis and antibiotic usage of any kind of infection.

In our study, more than half of the extrahepatic cases died, and only around $6 \%$ cases improved. But among the intrahepatic cases, $72.9 \%$ improved, $12.5 \%$ deteriorated, and $14.6 \%$ died. Significant clinical improvement was evidenced by decreasing jaundice, dark urine into normal color, pale stool became yellow, and gradual decrease in liver size from hepatomegaly state as well as a decreasing median total bilirubin, direct bilirubin, ALT, GGT, and alkaline phosphatase indicating a biochemical improvement at 2 years of follow-up.

Our study indicates that many complications with cholestasis could probably be avoided if we were able to intervene early. The rate of success in re-establishing bile flow is dependent on the age of the infant at the time of the surgery as well as on the experience of the surgeon [12]. The success rate could be as high as $80 \%$ if the surgery takes place at less than 30 to 45 days of age [12]. Less than $20 \%$ of the patients who undergo hepatic PE at older than 90 days achieve bile drainage [12, 28-32]. For the children with unsuccessful surgery, LT is essential to ensure long-term survival [33]. In the present study, only 6 cases went for KPE but all were unsuccessful evidenced by no resolution of jaundice with persistent pale stool. KPE was not done in majority of cases due to the age of the child or refusal by the patient party, which ended up with an unsuccessful surgery. Among all BA, most of the cases died or deteriorated with hepatic cirrhosis. A study from Malaysia reported a nearly 50\% unsuccessful KPE (death or deteriorated with major morbidity) [11]. An earlier study on Bangladesh showed that a 2-year survival with a native liver was as low as $36 \%$ and LT was not possible [34].

INH usually constitutes one third cases of NC [6]. However, recent studies showed a lower proportion due to wide range of diagnostic facilities [7]. Ghaffar et al. from Egypt found that $21 / 26$ babies with INH (80.76\%) were in the good prognosis group. There are two different categories of INH with different prognosis, sporadic, and familial. For sporadic cases prognosis is very good with a recovery rate of $60-80 \%$ while in familial cases the recovery rate is $20-40 \%$ [22]. Jacquemin et al. [35] and Venigalla et al. [6] observed a resolution of jaundice within 3.5 months and normalized liver enzyme within 1 year of age. In this study, INH constitutes $25 \%$ of all cholestasis and $41.8 \%$ of intrahepatic cholestasis. Among them, 16 (80\%) improved, 3 (15\%) deteriorated, and only 1 died.

The oral Valganciclovir is a promising drug for CMV infection [10, 36, 37]. In 84.6\% cases of CMV (with Valganciclovir) and 50\% cases of CMV with HSV (with Valganciclovir and Acyclovir) improved. The 2 cases (100\%) of toxoplasmosis died due to unavailability of drug (Sulfadiazine) and poor compliance.

An early diagnosis and intervention in $\mathrm{CC}$ through excision are a safe procedure with fewer complications [38, 39]. In the present study, surgery showed excellent results with a success rate of $100 \%$ for choledochal cysts without any complications.

Levothyroxine therapy was useful for congenital hypothyroidism and Down syndrome with hypothyroidism $[40,41]$. The present study also found the same results.

The early elimination of galactose from the diet may prevent further progression of Galactosemia [4, 42]. In the present study, early lactose free diet was introduced to $2(100 \%)$ galactosemia cases and both the child improved without any morbidity.

The two cases of PIBD, in this study, did not survive despite adequate medical treatment. A similar study on Iran also reported no improvement in the condition of all seven patients with PIBD despite adequate medical treatment [43]. LT led to the survival of 5 patients while the other patients who did not undergo LT died at 2 months of age. Undoubtedly, it reflects the necessity of LT facility that might be lifesaving for many children.

$\mathrm{NC}$ due to sepsis or other systemic infections can occur even in the post neonatal period, and patients may have isolated cholestatic jaundice without other manifestations of infection. Thus, a strong suspicion of systemic infections, especially UTI, should be kept in mind with NC [44]. With an appropriate antibiotic, our 1 case of UTI with sepsis improved successfully without any complications.

Typically, the patients with PFIC types 1 and 2 have low or normal GGT level and low cholesterol level and develop early cholestasis. Surgical methods like ileal exclusion and partial external biliary diversion may be effective temporarily but at the end, and LT is the answer [4]. Due to unavailability of LT, our 1 case with PFIC died of liver cirrhosis with failure. 


\section{Limitations of study}

The study was self-funded and hence the scope of the study reduced in some ways. First of all, the sample size of the study was small. As there are only two hospitals in Bangladesh who provide specialized treatment on pediatric gastroenterology, using a bigger sample was not possible. Second, genetic analysis could not be done and lastly the duration of follow ups was short. A longer period of follow-ups could come up with some more information regarding the children specially the survival time of the deteriorated children. Further, the diagnosis of biliary atresia in our study was not confirmed by intra-operative cholangiography due to ethical reasons of invasive procedure and radiation exposure, especially in advanced cases that were deemed inoperable. The biliary atresia score, as suggested by El-Guindi et al. [45], could not be considered as the study was conceptualized before this landmark study was published. Although, scarcity of adequate medical services inside the country (drugs, intrventions, surgery, and health education) do not allow this study to offer new medical information, the authors attempted to portray the etiology and outcome scenario of a country with scarce resources.

\section{Conclusions}

In our study, BA was the most common cause of extrahepatic while INH was the most common cause of intrahepatic cholestasis and prompt referral and early diagnosis as well as the etiology of $\mathrm{NC}$ were the main determinants of the outcome of the infants.

The age of admission, etiology, and presence of ascites are the predictors of outcomes. Early age of presentation, referral, and diagnosis results in a favorable outcome concerning the non-improved cases with delayed admission. Treatable etiology leads to a better outcome.

For both types of cholestasis, a delay in referral to an appropriate center remains as a major problem for the cholestatic infants like many parts of South Asia including India [26, 27, 46-49]. Wrong diagnosis (like "breast milk jaundice"), false reassurance by the appearance of pigmented stool, early hospital discharge, and parental refusal of a referral might play as the key factors for delayed hospital admission [47].

The overall outcome in Bangladesh is far from satisfactory due to delayed age at presentation (over 3 months compared to recommended age of less than 60 days). A LT facility could be life-saving for many children.

\section{Recommendations}

The screening program with stool color card could be a useful tool for avoiding the late referral as well as improving the outcome of patients with neonatal cholestasis in the next future as it was successful in some Asian countries [28, 29]. LT service in Bangladesh should be established as soon as possible, so that more children with end stage liver failure can receive life-saving surgery.

\section{Abbreviations}

NC: Neonatal cholestasis; BA: Biliary atresia; INH: Idiopathic neonatal hepatitis; CBC: Complete blood count; TORCH: Toxoplasma, Rubella, Cytomegalovirus, Herpes simplex; CMV: Cytomegalovirus; UTI: Urinary tract infection; a1 AT: Alfa 1 antitrypsin; CC: Choledochal cyst; PFIC: Progressive familial intrahepatic cholestasis; PIBD: Paucity of intrahepatic bile duct; ALP: Alkaline phosphatase; ALT: Alanine aminotransferase; AST: Aspartate aminotransferase; GGT: Gamma-glutamyl transferase; PT: Prothrombine time; USG: Ultrasonogram; HBS: Hepatobiliary system; HS: Hepatobiliary scintigraphy; MRCP: Magnetic resonance cholangiopancreatography; LB: Liver biopsy; PCR: Polymerase chain reaction; NGRS: Non-glucose reducing substance; FT4: Free thyroxine; TSH: Thyroid stimulating hormone; MCT: Medium chain triglyceride; UDCA: Ursodeoxycholic acid; KPE: Kasai hepatoportoenterostomy; ESLD: End-stage liver disease; LT: Liver transplantation; BSMMU: Bangabandhu Sheikh Mujib Medical University; CLD: Chronic liver disease; $\mathrm{PH}$ : Portal hypertension.

\section{Acknowledgements}

The authors are thankful to the reviewers for their thoughtful comments and suggestions that helped us to improve the paper.

\section{Authors' contributions}

SM conceived the study, participated in its design and coordination, and prepared the first draft of this paper. JG worked on the design of the study and performed the statistical analysis as well as interpretation of the results. MP took part in the liver biopsy procedure and processing of the biopsy specimen and preparing histo-pathological report. FT took part in the design, coordination, and helped in preparing the manuscript. SSA was involved in overall supervision of the study, as well as revised the manuscript critically for important intellectual content. The authors read and approved the final version of the paper.

Funding

The study is self-funded.

\section{Availability of data and materials}

The dataset used in the current study is available from the corresponding author on reasonable request.

\section{Declarations}

\section{Ethics approval and consent to participate}

Ethical clearance was taken from ethical review committee of Dhaka Shishu (Children) Hospital (No. Admin/1040/DSH/2021). Written informed consents were obtained from parents of individual participants at the time of admission.

\section{Consent for publication \\ Not applicable.}

\section{Competing interests}

The authors declare that they have no competing interests.

\section{Author details}

1Pediatric Gastroenterology, Hepatology \& Nutrition, Bangladesh Institute of Child Health, Dhaka Shishu (Children) Hospital, Sher-E-Bangla Nagar, Dhaka 1207, Bangladesh. ${ }^{2}$ Institute of Statistical Research and Training, University of Dhaka, Dhaka, Bangladesh. ${ }^{3}$ Bangladesh Institute of Child Health, Dhaka Shishu (Children) Hospital, Dhaka, Bangladesh. ${ }^{4}$ Department of Pediatrics, BIHS General Hospital, Diabetic Association of Bangladesh, Dhaka, Bangladesh. ${ }^{5}$ Pediatric Gastroenterology, Hepatology \& Nutrition, Bangladesh Institute of Child Health, Dhaka Shishu (Children) Hospital, Dhaka, Bangladesh.

Received: 16 June 2020 Accepted: 13 December 2021

Published online: 03 January 2022 


\section{References}

1. Fawaz R, Baumann U, Ekong U, Fischler B, Hadzic N, Mack CL et al (2017) Guideline for the evaluation of cholestatic jaundice in infants: joint recommendations of the North American Society for Pediatric Gastroenterology, Hepatology \& Nutrition and the European Society for Pediatric Gastroenterology, Hepatology and Nutrition. J Pediatr Gastroenterol Nutr 64(1):154-168

2. Pandita A, Gupta V, Gupta G (2018) Neonatal cholestasis: a Pandora's box. Clin Med Insights Pediatr 12:1-6

3. Gunaydin M, Cil ATB (2019) Cholestasis in baby and infant. EMJ 4(3):73-82

4. Feldman AG, Sokol RJ (2013) Neonatal cholestasis. Neoreviews 14(2):63-71

5. Ghazy RM, Khedr MA (2019) Neonatal cholestasis: recent insights. Egypt Pediatr Assoc Gaz 67:1-14

6. Venigalla S, Gourley GR (2004) Neonatal cholestasis. Semin Perinatol 28:348-355

7. Bhatia V, Bavdekar A, Mathai J, Waikar Y, Sibal A (2014) Management of neonatal cholestasis: consensus statement of the pediatric gastroenterology chapter of Indian Academy of Pediatrics. Indian Pediatr 51(203):210

8. Lippincott Williams \& Wilkins, Philadelphia (2004) Guideline for the evaluation of cholestatic jaundice in infants: recommendations of the North American Society for Paediatric Gastroenterology. Hepatol Nutr J Pediatr Gastroenterol Nutr 39:115-128

9. Najafi M, Khodadad A, Khatami GR, Farahmand F, Falahi GH, Motamed F (2006) Prevalence of different aetiology in neonatal cholestasis. Iran J Pediatr 16(3):289-294

10. Luthufdeen MIM, Waidyanatha S (2016) A study on cholestasis in infants less than six months of age presenting to Lady Ridgeway Hospital for children, Colombo. Sri Lanka J Child Health 45(1):34-37

11. Lee WS, Chai PF, Boey CM, Looi LM (2010) Aetiology and outcome of neonatal cholestasis in Malaysia. Singapore Med 5(15):434-439

12. Dani C, Pratesi S, Raimondi F, Romagnoli C (2015) Italian guidelines for the management and treatment of neonatal cholestasis. Ital J Pediatr 41:1-12

13. Suchy FJ (2004) Neonatal cholestasis. Pediatr Rev 25:388-396

14. Harpavat S, Finegold MJ, Karpen SJ (2011) Patients with biliary atresia have elevated direct/conjugated bilirubin levels shortly after birth. Pediatrics 128:e1428-e1433

15. Davis AR, Rosenthal P, Escobar GJ, Newman TB (2011) Interpreting conjugated bilirubin levels in newborns. J Pediatr 158:562e1-565e

16. Kianifar HR, Tehranian S, Shojaei P, Adinehpoor Z, Sadeghi R, Kakhki VR et al (2013) Accuracy of hepatobiliary scintigraphy for differentiation of neonatal hepatitis from biliary atresia: systematic review and meta-analysis of the literature. Pediatr Radiol 43:905-919

17. Moyer V, Freese DK, Whitington PF, Olson AD, Brewer F, Colletti RB et al (2004) Guideline for the evaluation of cholestatic jaundice in infants: recommendations of the North American Society for Pediatric Gastroenterology, Hepatology and Nutrition. J Pediatr Gastroenterol Nutr 39:115-128

18. Yang JG, Ma DQ, Peng Y, Song L, Li CL (2009) Comparison of different diagnostic methods for differentiating biliary atresia from idiopathic neonatal hepatitis. Clin Imaging 33:439-446

19. Sevilla A, Howman-Giles R, Saleh H, Trpezanovski J, Concannon R, Williams K et al (2007) Hepatobiliary scintigraphy with SPECT in infancy. Clin Nucl Med 32:16-23

20. Lee WS, Looi LM (2009) Usefulness of a scoring system in the interpretation of histology in neonatal cholestasis. World J Gastroenterol 15:5326-5333

21. Rastogi A, Krishnani N, Yachha SK, Khanna V, Poddar U, Lal R (2009) Histopathological features and accuracy for diagnosing biliary atresia by prelaparotomy liver biopsy in developing countries. J Gastroenterol Hepatol 24:97-102

22. Ghaffar TYA, Naghi SE, Faramawy AAE, Shehata AM (2014) Study on short term outcome of infantile cholestasis. J Den Med Sci 13(12):9-15

23. Suchy FJ, Balistreri WF, Heubi JE et al (1981) Physiologic cholestasis: elevation of the primary serum bile acid concentrations in normal infants. Gastroenterology 80(5):1037-1041

24. Urganci N, Cetinkaya F, Kalyoncu D, Cakir E, Yilmaz B (2012) Infants with cholestasis: diagnosis, management and outcome. Marmara Med J 25:83-86

25. Ali M, Gupta S, Zafar SMA, Rashid M, Husain MM, Rabbi H et al (2014) Initiation of liver transplantation in bangladesh: report on the first two successful cases. J Health Popul Nutr 32(4):696-700
26. Nahid KL, Qureshi NK, Mazumder W, Karim B (2015) Clinical characteristics, biochemical profile and etiology of cholestatic jaundice in Bangladeshi infants: tertiary care hospital experience. JDNMC 24(01):37-41

27. Karim B, Kamal M (2005) Cholestatic jaundice during infancy: experience at a tertiary-care center in Bangladesh. Indian J Gastroenterol 24:52-54

28. Lien TH, Chang MH, Wu JF, Taiwan Infant Stool Color Card Study Group et al (2011) Effects of the infant stool color card screening program on 5-year outcome of biliary atresia in Taiwan. Hepatology 53(1):202-208

29. Gu YH, Yokoyama K, Mizuta K, Tsuchioka T, Kudo T, Sasaki H (2015) Stool color card screening for early detection of biliary atresia and long-term native liver survival: a 19-year cohort study in Japan. J Pediatr 166(4):897-903

30. Serinet $M O$, Wildhaber BE, Broué $P$, Lachaux A, Sarles J, Jacquemin E et al (2009) Impact of age at Kasai operation on its results in late childhood and adolescence: a rational basis for biliary atresia screening. Pediatrics 123:1280-1286

31. Schreiber RA, Barker CC, Roberts EA, Martin SR, Alvarez F, Smith L et al (2007) Biliary atresia: the Canadian experience. J Pediatr 151:659-665

32. Nio M, Ohi R, Miyano T, Saeki M, Shiraki K, Tanaka K (2003) Japanese biliary atresia registry: five- and 10-year survival rates after surgery for biliary atresia: a report from the Japanese Biliary Atresia Registry. J Pediatr Surg 38:997-1000

33. Hartley JL, Davenport M, Kelly DA (2009) Biliary atresia. Lancet 374(9702):1704-1713

34. Davenport M, Kerkar N, Mieli-Vergani G, Mowat AP, Howard ER (1997) Biliary atresia: the King's College Hospital experience (1974-1995). J Pediatr Surg 32:479-485

35. Jacquemin E, Lykavieris P, Chaoui N, Hadchouel M, Bernard O (1998) Transientneonatal cholestasis: origin and outcome. L Pediatr 133:563-567

36. Shah I, Bhatnagar S (2012) Biliary atresia and cytomegalovirus and response to Valganciclovir. Indian Pediatr 49:484-486

37. Muller A, Hubinger AME, Brandhorst G, Heep A, Bartmann P, Franz AR (2007) Oral valganciclovir for symptomatic congenital cytomegalovirus infection in an extremely low birth weight infant. J Perinatol 28:74-76

38. Chang HM, Huang HH, Huand ES, Kau CL, Hsu HY, Lee CY et al (1992) Polymerase chain reaction to detect human cytomegalovirus in livers of infants with neonatal hepatitis. Gastroenterology 103:1022-1025

39. Nessa M, Khan S (2016) Experiences in the management of choledochal cyst in pediatric patients in a tertiary care center of Bangladesh armed forces. JAFMC Bangl 12(2):117-121

40. Bhowmik A, Biswas T (2016) Isolated central hypothyroidism presenting with severe cholestasis, hepatosplenomegaly, and pallor: a case report and review of literature. J Clin Neonatol 5(3):202-204

41. Mantri R, Bavdekae SB, Save SU (2016) Congenital hypothyroidism: an unusual combination of biochemical abnormalities. Hindawi Publishing Corporation. https://doi.org/10.1155/2016/2678578

42. Beltran MA, Alvarez M, Palazon SC (2007) Galactosemia as a cause of neonatal ascitis and cholestasis. Pediatria Catalana 67(5):235-240

43. Mozhgan Z, Bita G, Mahmood H, Hajar E (2013) Paucity of intrahepatic bile ductsin neonates: the first case series from Iran. Iran J Pediatr 23(1):65-70

44. Maria N, Pereira D, Shah I (2017) Neonatal cholestasis mimicking biliary atresia: could it be urinary tract infection? SAGE Open Med Case Rep 5:1-3

45. El-Guindi MAS, Sira MM, Sira AM, Salem TAM, El-Abd OL, Konsowa HAS et al (2014) Design and validation of a diagnostic score for biliary atresia. J Hepatol 61(1):116-123

46. Tiker F, Tarcan A, Kilicdag B, Gurakan et al (2006) Early onset conjugated hyperbilirubinemia in newborn infants. Indian J Pediatr 73(5):409-412

47. Yachha SK, Mohindra S (1999) Neonatal cholestasis syndrome: Indian scene. Indian J Pediatr 66(Suppl 1):94-96

48. Hamid F, Afroza A, Ray PC (2012) Aetio-clinical profile of cholestatic jaundice during infancy-study of 30 cases in tertiary care hospital. Bangladesh Med J 41(2):34-39

49. Matthai J, Paul S (2001) Evaluation of cholestatic jaundice in young infants. Indian Pediatr 38:893-898

\section{Publisher's Note}

Springer Nature remains neutral with regard to jurisdictional claims in published maps and institutional affiliations. 